Background: Rheumatoid arthritis (RA) is categorized as seropositive or seronegative referring to the presence or absence of IgM rheumatoid factor (IgM-RF) and/ or anti-citrullinated protein antibodies (ACPA).

Patients with an autoimmune disease are more likely to develop additional autoimmune conditions than individuals without pre-existing autoimmune disease. Objectives: The aims of this study were to examine patterns of polyautoimmunity in seropositive compared with seronegative patients with recent-onset RA. Methods: The study was register-based and used the nationwide DANBIO register (identification of recent-onset ( $<1$ year) seropositive, ICD-10 M05 and seronegative, M06, RA patients) linked to the Danish National Patient Registry and the Danish National Prescription Registry to obtain information on additional autoimmune diseases (see Table 1 for autoimmune conditions). Using age and sex adjusted logistic regression analysis, the odds ratio (OR) of prevalent polyautoimmunity in seropositive compared with seronegative patients at the time of RA diagnosis was calculated.

To estimate the hazard ratio $(\mathrm{HR})$ for developing yet another autoimmune disease in the 5 years after RA diagnosis, adjusted cause-specific Cox regression models were performed. Several sensitivity analyses were carried out including alternative exposure and outcome definitions.

Results: In total, 12,517 patients with recent-onset RA were included. The groups were similar in terms of disease characteristics and DMARD treatment, but seropositive patients were younger and included more women, see Table 1. Patients with seropositive RA had an OR of 0.79 (95\% Cl 0.71-0.89) for baseline presence of polyautoimmunity compared with seronegative patients, whereas the 5 -year HR was $0.86(95 \% \mathrm{Cl} 0.71-1.05)$ for incident polyautoimmunity. The results remained similar in all sensitivity analyses.

Conclusion: Patients with seropositive RA had a slightly lower prevalence and incidence of polyautoimmunity compared to seropositive patients. The results were somewhat surprising, yet very robust, and thus raises the question if seronegative RA is in fact "more autoimmune" despite the absence of (identified) autoantibodies.

Table 1. Characteristics, prevalence, and incidence of polyautoimmunity in incident RA patients.

\begin{tabular}{lcc}
\hline & Seropositive & Seronegative \\
\hline $\mathrm{N}$ & 7983 & 4534 \\
Age in years & $59.4(48.6$ to 69.1$)$ & $63.0(51.5$ to 72.3$)$ \\
Women & $5476(69 \%)$ & $2856(63 \%)$ \\
HAQ-DI & 0.857 & 0.875 \\
& $(0.375$ to 1.375$)$ & $(0.375$ to 1.375$)$ \\
DAS28-CRP & $4.3(3.3$ to 5.2$)$ & $4.6(3.6$ to 5.5$)$ \\
CRP, mg/mL & $10(4$ to 23$)$ & $10(3$ to 26$)$ \\
VAS physician, 0-100mm & $27(15$ to 45$)$ & $30(16$ to 47$)$ \\
Treated with methotrexate & $86 \%$ & $84 \%$ \\
-other csDMARD, \% & $24 \%$ & $24 \%$ \\
-Biological DMARD, \% & $5 \%$ & $6 \%$ \\
Smoking status: & $13 / 11 / 17 / 59 \%$ & $9 / 9 / 23 / 59 \%$ \\
Current / previous / never / unknown, \% & $549(10.6 \%) /$ & $349(12.8 \%) / 1.5$ \\
Prevalence of polyautoimmunity / & $1.4 \% / 6.7 \% / 1.4 \%$ & $\% / 7.8 \% / 1.5 \%$ \\
diabetes mellitus type 1 / autoimmune $\quad$ & \\
$\quad$ thyroid disease / inflammatory bowel disease & $0.79(0.71$ to 0.89$)$ & 1 (ref.) \\
Age and sex adjusted odds ratio & $373(4.7 \%)$ & $242(5.3 \%)$ \\
Incident cases of polyautoimmunity & $0.86(0.71$ to 1.05$)$ & 1 (ref.) \\
Adjusted hazard ratio & &
\end{tabular}

Continuous variables shown as median with interquartile ranges. Autoimmune conditions included:autoimmune thyroid disease (redeemed prescriptions of $\mathrm{ATC}=\mathrm{H} 03 \mathrm{~A}$ ); diabetes mellitus type 1 (ICD-10 diagnosis E10 combined with redeemed prescriptions of insulin, ATC $=$ A10A); pernicious anaemia (D51.0); autoimmune haemolytic anaemia (D59.1); idiopathic thrombocytopenic purpura (D69.3); Autoimmune adrenalitis (E27.1B); multiple sclerosis (G35); neuromyelitis optica (G36.0); Guillain-Barré syndrome (G61.0); myasthenia gravis (G70); inflammatory bowel disease (K50-1); primary biliary cirrhosis (K74.3); primary sclerosing cholangitis (K83.0); autoimmune hepatitis (K75.4); celiac disease (K90.0); pemphigus vulgaris (L10.0); bullous pemphigoid (L12.0); dermatitis herpetiformis (L13.0); alopecia areata (L63); vitiligo (L80); lichen sclerosis (L90.0); chronic interstitial cystitis (N30.1).

Acknowledgements: We acknowledge all patients and all Danish departments of rheumatology contributing to the DANBIO registry.

Disclosure of Interests: Amalie Hagelskjær: None declared, René Cordtz: None declared, Sofie Bliddal: None declared, Anders Sandermann Mortensen: None declared, Salome Kristensen: None declared, Claus Henrik Nielsen: None declared, Ulla Feldt-Rasmussen: None declared, Christian Torp-Pedersen: None declared, Lene Dreyer Grant/research support from: Grants from BMS, Galderma, and Eli Lilly DOI: 10.1136/annrheumdis-2021-eular.668

\section{POS0314 \\ PROSPECTIVE EVALUATION OF A DEDICATED, SERIAL OCCUPATIONAL THERAPY EXERCISE PROGRAM ON HAND FUNCTION IN RHEUMATOID ARTHRITIS (RA)}

W. Pietrowski ${ }^{1}$, K. Richter-Bastian ${ }^{2}$, K. Storck-Müller ${ }^{2}$, U. Müller-Ladner ${ }^{1}$, U. Lange ${ }^{1}$, I. Tarner ${ }^{1} .{ }^{1}$ Campus Kerckhoff, Justus-Liebig-University Gießen,
Department of Rheumatology, Clinical Immunology, Osteology and Physical Medicine, Bad Nauheim, Germany; ${ }^{2}$ Rheumazentrum Mittelhessen, Department of Rheumatology, Bad Endbach, Germany

Background: Rheumatoid arthritis (RA) frequently affects the joints of the hands causing severe pain and significant functional impairment affecting all activities of daily living. At early stages, functional impairment is primarily caused by reversible joint and tendon sheath effusions while at later stages synovial thickening, muscular atrophy and irreversible damage to cartilge, bone, tendons and ligaments cause a reduced range of motion and deformities. Occupational therapy aims to maintain and improve joint mobility and function. However, there is limited data on the effectiveness of serial exercise programs specifically aiming at maintaining and improving hand function in RA.

Objectives: To prospectively evaluate the effects of a dedicated exercise program on hand function, grip strength, joint mobility and pain compared with conventional outpatient physiotherapy not specifically targeting hand function.

Methods: A total of 51 RA patients receiving outpatient physiotherapy once a week were enrolled and randomized to either continue their usual therapy (control group) or to participate in a dedicated hand function training (HFT) twice a week in addition to their usual therapy (HFT group). The HFT program was supervised by an occupational therapist who conducted once weekly HFT sessions and instructed the participants to perform an additional self-administered HFT session at home once a week. Study duration was six months with an option to continue HFT for additional 6 months. Study participants were evaluated at baseline and every 3 months.

The primary outcomes hand function and grip strength were assessed using standardized questionnaires (Cochin hand function scale (CHFS), Australian/ Canadian Osteoarthritis Hand Index (AUSCAN), Michigan Hand Outcomes Questionnaire (MHOQ)) and dynamometer/pinch gauge readings for different types of grip (cylindrical grasp, spherical grasp, hook grasp, tip to tip pinch and key pinch), respectively. The secondary outcomes joint mobility and pain were assessed using a digital goniometer and visual analogue scales (VAS), respectively. Statistical analysis used the Wilcoxon test for evaluating changes of parameters over time within the groups and the Kruskal Wallis test for group comparisons and Bonferroni-Holm correction.

Results: 41 of the 51 patients (HFT group: $n=20$, mean age $60.3 \pm 8.4$ years; control group: $n=21$, mean age $60.5 \pm 11.5$ years) completed the 6 -month study period, 14 patients extended their HFT to 12 months. Within the 6-month study period there were no significant improvements of the primary outcome parameters and of pain within the groups vs. baseline and no significant differences between the groups. Only the CHFS improved significantly in the HFT group as of month 9. A significant improvement of the range of motion vs. baseline was achieved by HFT as early as 3 month after baseline which persisted up to month 12. As some improvement was also seen in the control group, there was no significant difference between the groups. Hand mobility improved particularly in the subgroup of patients $>60$ years of age. There was no influence by pain intensity at baseline (VAS $\leq 50 \mathrm{~mm}$ vs. VAS $>50 \mathrm{~mm}$ )

Conclusion: Serial dedicated HFT for 6 months resulted in improved joint mobility but did not improve global hand function, grip strength and pain in RA patients. A longer treatment duration ( $\geq 12$ months), a higher treatment frequency ( $>2$ times/week) and a larger cohort of patients may be required.

Disclosure of Interests: None declared

DOI: 10.1136/annrheumdis-2021-eular.3806

\section{Scleroderma, myositis and related syndromes}

\section{POS0315 LONG-TERM SAFETY AND EFFICACY OF LENABASUM DURING 3 YEARS IN AN OPEN-LABEL EXTENSION (OLE) OF A PHASE 2 STUDY OF LENABASUM IN REFRACTORY SKIN DISEASE IN DERMATOMYOSITIS (DM)}

V Werth. ${ }^{1}$, J. Concha ${ }^{1}$, J. Burroughs ${ }^{1}$, J. Okawa ${ }^{1}$, R. Feng ${ }^{1}$, A. Jobanputra ${ }^{1}$, R. Borucki ${ }^{1}$, K. Hally ${ }^{2}$, E. Hejazi ${ }^{1}$, M. Tillinger ${ }^{2}$, S. Constantine ${ }^{2}$, N. Dgetluck ${ }^{2}$, B. White ${ }^{2}$. 'University of Pennsylvania, Dermatology, Philadelphia, United States of America; ${ }^{2}$ Corbus Pharmaceuticals, Clinical, Norwood, United States of America

Background: Lenabasum is a synthetic, non-immunosuppressive, selective cannabinoid receptor type 2 agonist that activates resolution of inflammation. Lenabasum had acceptable safety and tolerability and improved efficacy outcomes in the initial 16-week double-blinded, randomized, placebo-controlled Part A of Phase 2 trial JBT101-DM-001 (NCT02466243) in DM subjects with refractory skin involvement. In that study, lenabasum or placebo was added to stable background treatment, with immunosuppressive therapies allowed.

Objectives: To assess long-term safety and efficacy in DM subjects in this study. Methods: Subjects who completed Part A of the Phase 2 study $(n=22)$ were eligible to receive oral lenabasum $20 \mathrm{mg}$ BID in an open-label extension (OLE) that assessed safety and efficacy at 4 weeks, then every 8 weeks. 